\title{
Anaesthesia for cesarian section in a patient with portal hypertension caused by Schistossomiasis. Case report
}

\section{Carvalho, VH ${ }^{1}$; Scherner, B ${ }^{1}$; Picoli , V ${ }^{1}$; Shimabucoro, $\mathrm{S}^{1}$; Ferreira, $\mathrm{V}^{1}$; Braga, $\mathrm{A}^{1}$}

1. State University of Campinas - São Paulo - Brazil

Background: Schistosomiasis (Katayama fever) results from infection with the parasitic flatworms Schistosoma mansoni. It is a common infection in tropical countries where bodies of water are infested with snails of the Biomphalaria genera (intermediate host). The disease affects mainly children and childbearing woman who cook, wash clothes or work near contaminated lakes or rivers. Pregnant woman of endemic regions may have chronic or acute forms of disease ${ }^{1,2}$. The liver disfunction can cause coagulation disorders and bleeding, usual complications that can worsen obstetric risk.

Case report: DSS, age $30,73 \mathrm{~kg}$ of weight and $150 \mathrm{~cm}$ of height, BMI 32,4 kg/m2, physical status ASA III, 36 weeks of gestational age, with a previous diagnosis of cirrhosis with portal hypertension due to schistosomiasis. The patient had recent episodes of oropharingeal hemorrhage caused by esophageal varices. As fetal monitoring showed recurrent signs of fetal distress, the obstetric team decided to interrupt the pregnancy at once by cesarian section. She had a previously obstetric history (7 years earlier) of urgent Csection at 36 weeks due to fetal distress and intense oral bleeding when general anesthesia was performed for the birth of her first child. At that time, she was diagnosed and treated for Schistosomiasis with praziquantel. Actual physical exam without significant alterations, except for mild anemia, Mallampatti 3. In use of propranolol and ferrous sulphate.

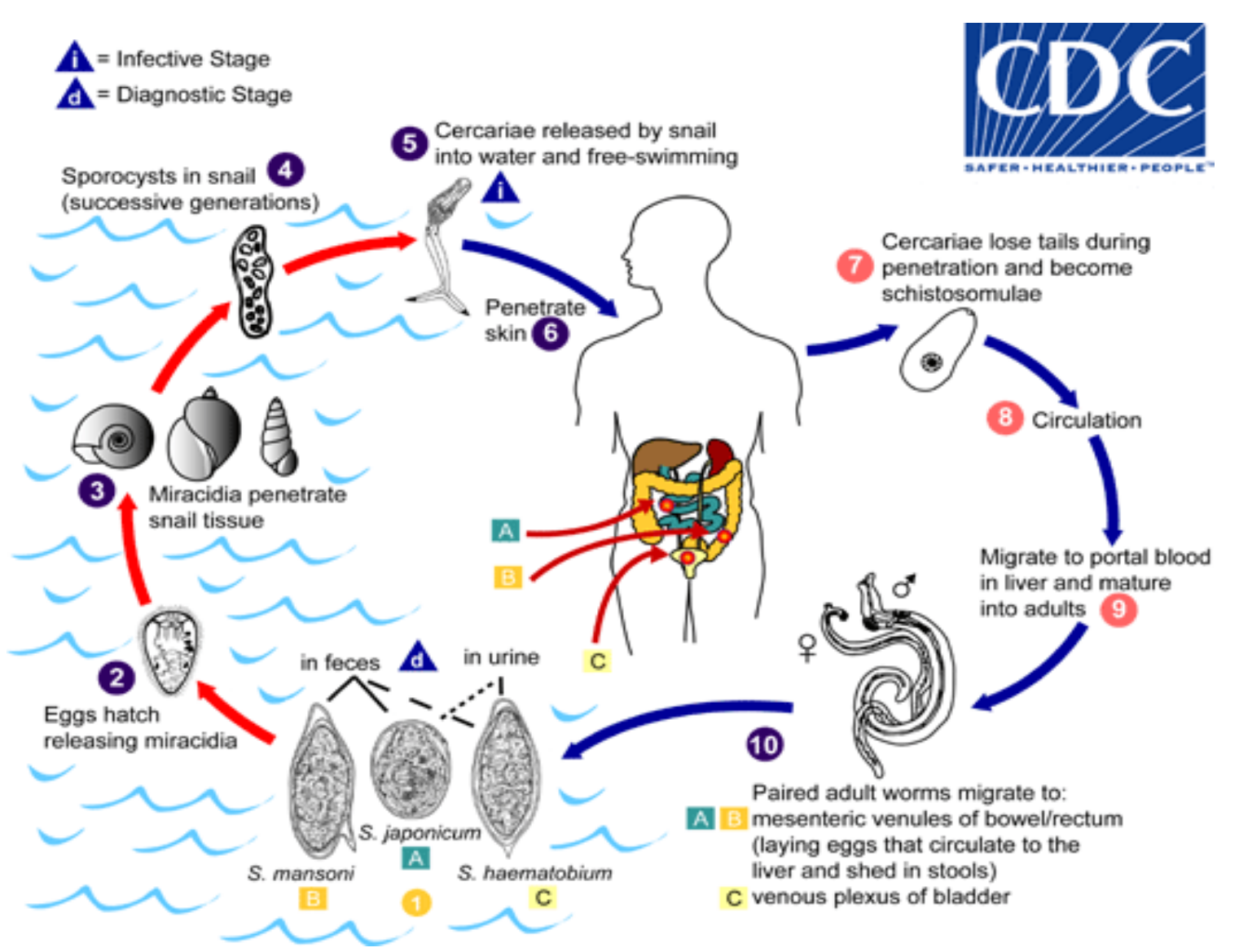

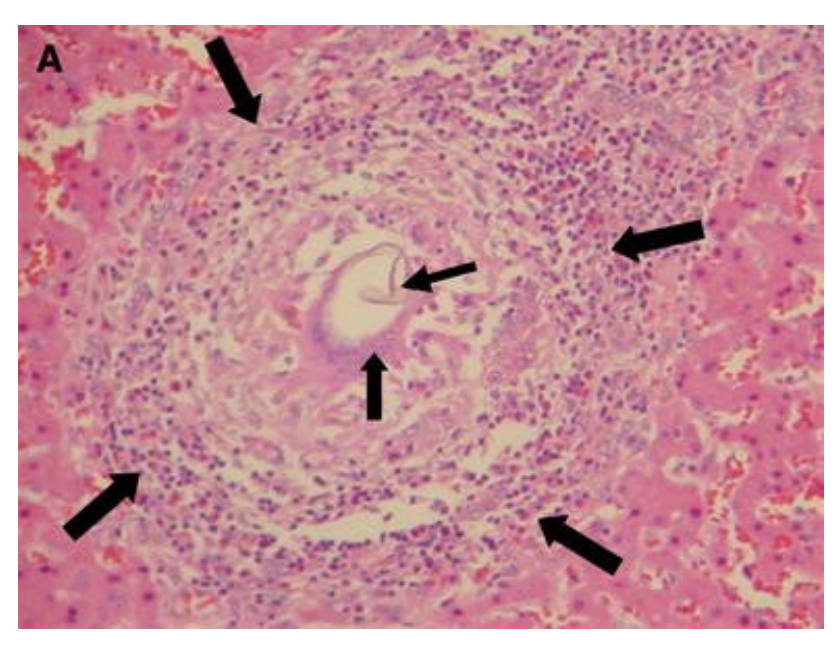

Egg

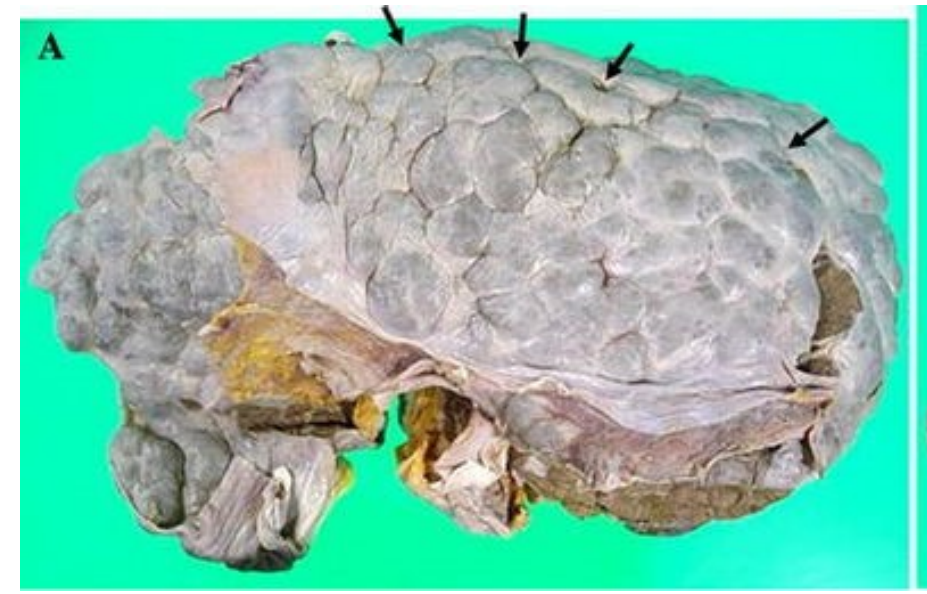

Mimicking "turtle-back"

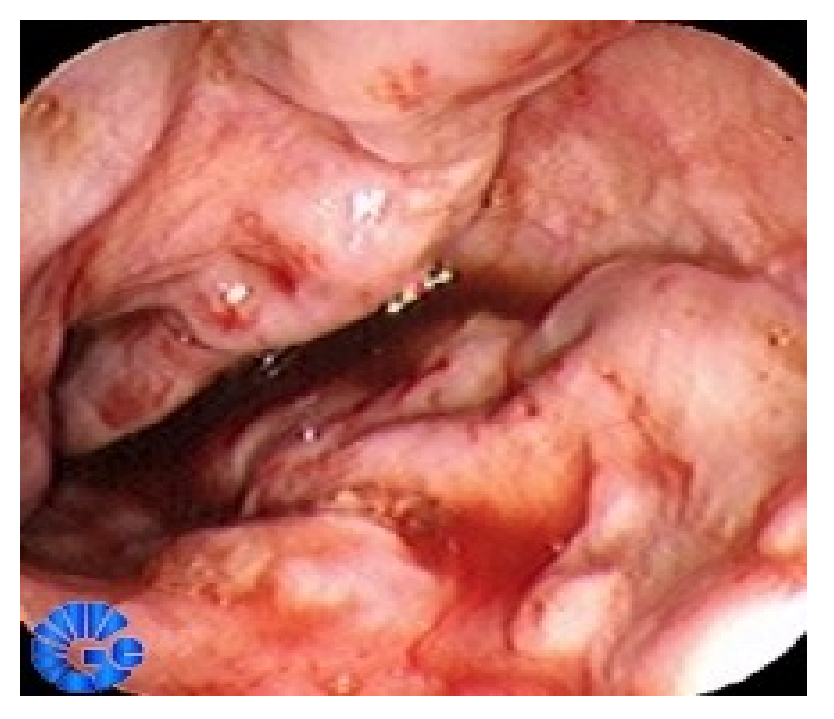

Esophageal varices

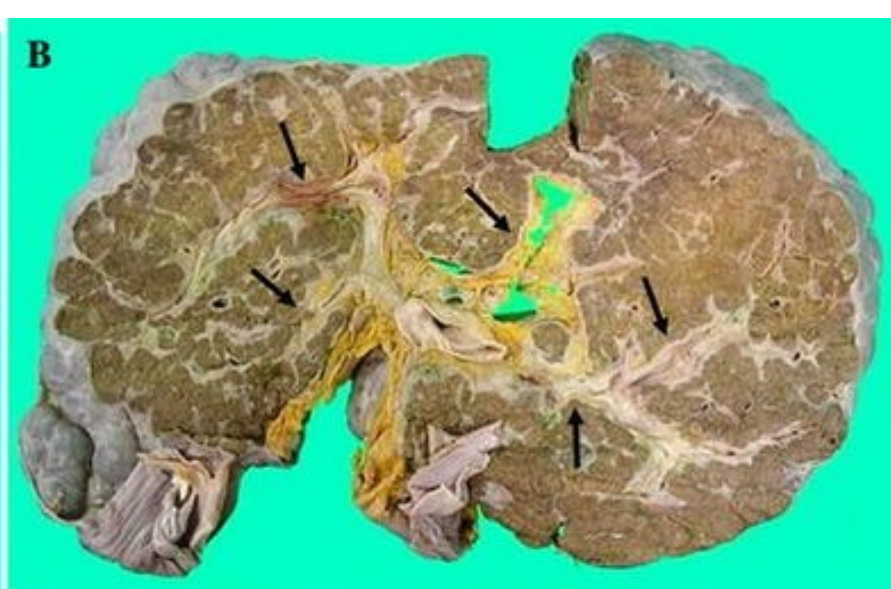

Severe fibrous thickening of the portal vein branchs in the central part
The abdominal echography showed signs of liver cirrhosis, portal hypertension with splenomegaly. Digestive Endoscopy evidenced multiple esophageal varices with signs of recent bleeding. Coagulation tests were at normal range, except platelet count: 73.000. Liver laboratory tests were normal.

We indicated spinal anestesia with $27 \mathrm{G}$ needle, $12 \mathrm{mg}$ of heavy bupivacaine $0,5 \%, 20 \mathrm{mcg}$ fentanyl and $60 \mathrm{mcg}$ morphine. Standard monitoring, a large venous peripheral cateter and urinary output was done. The surgery was devoid of complications. Apgar:10 and 10. The anaesthetic tecnique was chosen based on clinical manifestatons, lack of laboratory exams that would contraindicate a neuroaxial block and avoiding the need of a difficult airway management in a pregnant woman with general anaesthesia. The risk of spinal or general anaesthesia have to be individualized and informed consent must be obtained.

\section{References:}

1.Friedman JF, Mital P, Kanzaria HK, Olds GR, Kurtis JD. Schistosomiasis and pregnancy. Trends Parasitol. 2007; 23(4): 159-64.

2. Lauretti G R, McKay RSF. Infectious diseases in pregnancy. In: Obstetric anesthesia and uncommon disorders. 2nd edition. 2011:332-333.

3. Katz D, Beilin Y. Disorders of coagulation in pregnancy. BJA 2015; 115 (2): 75-88. 